\title{
Un pie en el cielo y otro en la ciencia. Las interacciones productivas de Miquel Crusafont (1910-1983)
}

\section{Carlos Acosta Rizo (*)}

$\left(^{*}\right) \quad$ Centre d'Estudis de Història de la Ciència, CEHIC. Universitat Autònoma de Barcelona geo_acosta@hotmail.com

SUMARIO: 1.-Introducción. 2.-Emergencia y pervivencia científica de Miquel Crusafont. 2.1.-Creación de redes profesionales. 2.2.—Estrategias de conciliación y ambivalencia. 3.—La Institucionalización. De la Sección de Paleontología del Museo de Sabadell al Institut Català de Paleontología (ICP). 4.-Conclusiones.

RESUMEN: Pasadas la Guerra Civil y la II Guerra Mundial (incluso durante ella) algunos científicos españoles - bajo condiciones nada favorables - reemprendieron la divulgación de su quehacer científico y del patrimonio paleontológico español. Ambas cosas interesaron a los investigadores extranjeros y algunos de ellos interactuaron con aquéllos en las décadas siguientes. Uno de estos personajes fue el paleontólogo catalán Miquel Crusafont Pairó (19101983) quien desplegó una intensa actividad profesional cuyos resultados le permitieron tanto afianzarse en el ámbito científico nacional como lograr el reconocimiento internacional por parte de colegas de diversa ideología científica y, de paso, posicionar a la Paleontología española en Europa. Este artículo analiza algunas de las estrategias desplegadas por Crusafont para sortear distancias espaciales e ideológicas, y describe sus actuaciones particulares en torno a la defensa y gestión de yacimientos y colecciones, y a la institucionalización de su ámbito de trabajo. Todo ello le permitió construir una trayectoria investigadora desde la provincia de una región periférica con las condiciones particulares de aquella España, y sentar las bases de la Paleontología y la Paleoantropología en la Cataluña contemporánea.

PALABRAS CLAVE: Paleontología, Paleoantropología, Miquel Crusafont, finalismo, neodarwinismo.

KEY WORDS: Palaeontology, Palaeoanthropology, Miquel Crusafont, Finalism, Neo-Darwinism. 


\section{Introducción $(*)$}

Miquel Crusafont Pairó (1910-1983) se licenció en farmacia en 1933, en la Universidad de Barcelona, pero ya había publicado su primer trabajo paleontológico en $1932^{1}$. Él mismo define aquellos años como una etapa de vocación mal definida o «etapa preparatoria» ${ }^{2}$. Según su hijo, «els estudis de farmàcia, fets amb poca brillantor, pretenien assolir per a la família una estabilitat econòmica, però no responien en absolut a una vocació» ${ }^{3}$. Crusafont atribuye a su descubrimiento del yacimiento de Can Llobateres (en 1926) - realizado conjuntamente con Ramon Arquer Costajussà (19091991) - gran peso en el desarrollo de su vocación científica, hasta ese momento más orientada al excursionismo, la arqueología y la mineralogía ${ }^{4}$.

Gracias a que su mujer, Júlia Sabaté, quien llevaba buena parte del negocio familiar (conformado por dos farmacias), Crusafont pudo dedicar tiempo a la investigación. Su vocación y la necesidad de ser aceptado entre naturalistas y paleontólogos, alguno de los cuales había insinuado su condición de amateur, lo llevaron de nuevo a la universidad para licenciarse en ciencias naturales en la Universidad de Madrid en 1948 y doctorarse en la misma en $1950^{5}$.

El resultado científico de su labor fue un incremento extraordinario del registro paleontológico de los mamíferos y un conocimiento más preciso de la estratigrafía del Terciario español. Atraído por los métodos biométricos,

(*) Esta investigación ha sido realizada en el marco del proyecto Servei d'Arxius de Ciència (SAC) del Centre d'Història de la Ciència (CEHIC) de la Universitat Autònoma de Barcelona, que ha permitido catalogar cerca de 12.000 de los aproximadamente 15.000 documentos que conforman la sección epistolar del fondo documental del Archivo Miquel Crusafont del Institut Català de Paleontologia en Sabadell. Puede consultarse en línea: www.sac.cat.

1. Fernández de Villalta, Josep; Crusafont, Miquel. Primera nota sobre vertebrats fòssils miocènics del Vallès: la presència del Deinotherium giganteum Kaup var. laevius Jourdan a Sant Quirze de Galliners. Butlletí de la Institució Catalana d'Història Natural. 1933; 33 (4-5): 258-261.

2. Crusafont Pairó, Miquel. Quaranta anys de Paleontologia al carrer de Sant Antoni. Arraona. 1981; 12, II època, tardor: 37-38 (38).

3. Crusafont Sabaté, Miquel. L'entorn familiar d'en Miquel Crusafont Pairó. Quadern de les arts i lletres de Sabadell. 1984; monogràfic 38: 72-75.

4. Crusafont Pairó, n. 2, p. 37-38.

5. Crusafont estaba más y mejor relacionado con la academia madrileña, representada por personajes como Eduardo Hernández Pacheco (1872-1965) y Bermudo Meléndez (1912-1999) que con la barcelonesa, representada por Lluís Solé Sabarís (1908-1985) y Josep Ramon Bataller (1890-1962). Quizás eso explica que cursase sus estudios en la capital y no en la Universidad de Barcelona. 
se interesó por la posibilidad de expresar cuantitativamente los cambios morfológicos interespecíficos observables en piezas esqueléticas. Fue principal divulgador en España del tema de la evolución (y de la Paleontología en general), difusión orientada hacia el posicionamiento del paleontólogo como baluarte del estudio del proceso evolutivo, y hacia la interpretación finalista del fenómeno de la vida y de la hominización.

No se pretende aquí hacer una biografía del personaje ${ }^{6}$ sino servir de lente de aumento a otras historiografías que se entrecruzan en este volumen, identificando y analizando algunas de las estrategias que utilizó para emerger y pervivir científicamente haciendo paleontología y hablando de evolución ${ }^{7}$. También se intenta mostrar cómo logró apoyarse y destacar en un grupo científico afín a sus conceptos; cómo evitó desprenderse de las corrientes novedosas pero antagónicas a su ideología; y, finalmente, delinear las intenciones y las motivaciones que lo guiaron en el proceso de apuntalar institucionalmente su labor científica en torno a la paleobiología.

\section{Emergencia y pervivencia científica de Miquel Crusafont}

Al inicio de la España franquista y durante la Segunda Guerra Mundial, el aislamiento era un problema para los investigadores españoles ${ }^{8}$. Crusafont

6. Para una biografía más detallada véase: Mañosa, Montserrat. Miquel Crusafont i Pairó (Sabadell, 1910-1983). L'escola paleontològica de Sabadell. In: Camarasa, Josep Maria; Roca Rosell, Antoni, ed. Ciència i tècnica als Països Catalans: Una aproximació biogràfica. Barcelona: Fundació Catalana per a la Recerca; 1995, p. 1445-1472; Amics de les Arts i les Lletres de Sabadell. Quadern de les arts i lletres de Sabadell. 1984; monogràfic 38.

7. Contrariamente a lo que se pueda pensar, la carga materialista que la evolución podía imponerle a la paleontología no fue obstáculo para que ésta fuese rápidamente integrada al CSIC, a la sombra de la geología y de la mano de una supuesta afinidad del colectivo de geólogos con el régimen. Para una historia de la paleontología española en el siglo XX véase: Truyols, Jaume. Desarrollo histórico de la paleontología contemporánea en España. In: Historia de la Paleontología. Madrid: Real Academia de Ciencias Exactas, Físicas y Naturales; 1988, p. 45-68; Perejón, Antonio. Aproximación a la historia de la Paleontología española. Enseñanza de las Ciencias de la Tierra. 2001; 9 (2): 127-143.

8. En trabajos recientes el aislamiento se analiza de manera general para otras disciplinas y para la universidad: Otero Carvajal, Luís. E., dir. La destrucción de la ciencia en España. Depuración universitaria en el franquismo. Madrid: Universidad Complutense de Madrid; 2006; Claret Miranda, Jaume. El atroz desmoche. La destrucción de la Universidad española por el franquismo, 1936-1945. Barcelona: Crítica; 2006. Para el caso particular de la paleontología véase: Pelayo, Francisco. Debatiendo sobre Darwin en España: Antidarwinismo, teorías evolucionistas, alternativas y síntesis moderna. Asclepio. 2009; 61 (2): 101-128 (101-102). 
intentó resolverlo creando redes profesionales a través del contacto epistolar y personal, y utilizando convenientemente la conciliación y la ambivalencia.

Desde el Museo de Sabadell escribió a centros de investigación paleontológica, bibliotecas, museos y paleontólogos de todo el mundo, pidiendo información y adjuntando sus publicaciones, por lo general realizadas en colaboración con Joan Andreu Rovira (1916-1991), Jaume Truyols Santoja (n. 1921) y Josep Fernández de Villalta (1913-2003) ${ }^{9}$.

A pesar de ello, Crusafont se quejaba del aislamiento, señalando que contaba con muy pocos contactos extranjeros, entre los que destacaba a Jean Viret (1894-1970) del Museo de Ciencias Naturales de Lyon, y George Gaylord Simpson (1902-1984) del Museo Americano de Historia Natural de Nueva York, a quien dice estar aislado y no poder consultar sobre la evolución con personas mejor formadas. Crusafont también atribuía el no ser citado al hecho de que su trabajo, publicado por el Museo de Sabadell, no tenía difusión alguna. Por ejemplo, consideró que ese era el motivo por el cual no había sido invitado a participar en un monográfico que se preparaba sobre la evolución en Arbor: «¿Es que tengo que conformarme a que lo que hago no pueda traspasar la órbita de mi ciudad?» ${ }^{10}$.

\subsection{Creación de redes profesionales}

Conforme Crusafont publicaba y remitía sus estudios, sentía la necesidad de trabajar en equipo «i, si fos possible en [col-laboració] internacional perquè intuïem que era aquest el veritable camí de treball en aquelles èpoques d'ultraespecialització» ${ }^{11}$. En 1946 empieza a viajar por Europa financiado por la Junta de Relaciones Culturales del Ministerio de Asuntos

9. En los años cuarenta Crusafont publicó en ediciones especiales del Museo de Sabadell, de la Fundació Bosch i Cardellach y en el Boletín del Instituto Geológico y Minero de España. A partir de 1956 publicó preferentemente en los medios que él creó y dirigió, el Boletín Informativo de las Actividades Europeas en Paleontología de Vertebrados (1956-1968) y Fossilia (1965-1969) del Museo de Sabadell y, desde 1969, en el Boletín Informativo del Instituto Provincial de Paleontología.

10. Crusafont, M. Copia de carta a G. Simpson. 11 May 1948. Archivo Miquel Crusafont (en adelante, AMC) doc . 045b/Cprof/4723; Crusafont, M. Copia de carta a Bermudo Meléndez. 15 Dic 1950, AMC, doc. 045c/Cprof/4844.

11. Crusafont, n. 2, p. 38-39. 
Exteriores, propuesto por el $\mathrm{CSIC}^{12}$, para establecer contacto personal con sus corresponsales, especialmente con franceses y suizos, entre los que se cuentan, además de Viret, a Colette Deschaseaux, Jean Piveteau (18991991) y Robert Hoffstetter (1908-1999) en el Laboratorio de vertebrados y Paleontología Humana de la Universidad de Paris VI; Léonard Ginsburg (1927-2009), Jean-Pierre Lehman (1914-1981) y René Lavocat (1909-2007) en el Laboratorio de Paleontología del Museo Nacional de Historia Natural de París; y Samuel Schaub (1882-1955) y Johannes Hürzeler (1908-1995) en el Museo de Historia Natural de Basilea. Fue con Lavocat con quien Crusafont discutió por primera vez el asunto de la evolución en el sentido finalista, «passejant pel Jardí de Paltes de París» ${ }^{13}$. Según el mismo Crusafont escribe:

«Aquest viatge ens permetré d'estrènyer relacions internacionals que aviat augmentarien qui-sap-lo [...] Es pot dir que l'any 1946 posàrem els fonaments d'aquesta colllaboració que, com es por suposar, fou després àmplia i molt fecunda ${ }^{14}$.

Los paleontólogos extranjeros también llegaron a España, inicialmente para participar en los eventos que Crusafont organizó en los años cincuenta y sesenta, especialmente los Cursillos Internacionales de Paleontología ${ }^{15}$ (Figura 1).

12. Se conservan sus cartas a varios investigadores del CSIC sobre los resultados de este viaje. Crusafont, M. Copias de cartas a: José Albareda. 28 Ene 1946, AMC, doc.045b/CProf/4502; Primitivo Hernández Sampelayo. 6 Feb 1946, AMC, doc. 045b/CProf/4507; Eduardo Hernández Pacheco. 6 Feb 1946, AMC, doc. 045b/CProf/4508. Albareda, José. Carta a M. Crusafont. 11 Feb 1946, AMC, doc. 045b/CProf/4509.

13. Truyols, Jaume. L'obra científica del doctor Miquel Crusafont i Pairó (1910-1983). Butlletí de la Institució Catalana d'Història Natural. 1986; 53: 19-36 (35). Crusafont partió inicialmente de una base neolamarckista, pero pronto encontró afinidad en el finalismo (base filosófica de la ortogénesis), reafirmado al descubrir a Teilhard de Chardin; Colomer, Eusebi. Miquel Crusafont i Pierre Teilhard. Quadern de les arts i lletres de Sabadell. 1984; monogràfic 38: 56-59; Agustí, Jordi. L'evolucionisme meridional de Miquel Crusafont. Revista d'Història. 1994; 14: $29-43$.

14. Crusafont, n. 2, p. 38-39. Crusafont ofreció conferencias en las universidades de París, Montpellier, Dijon, Utrecht, Ámsterdam, Leyden, Florencia, Berlín, Mainz, Lyon, Ferrara, Uppsala y Helsinki, y en los museos de Historia Natural de Darmstadt, Weimar y Basilea. También participó en coloquios internacionales, por ejemplo, los de Paleontología de vertebrados en París, patrocinados por la Fundación Rockefeller y el Centre National de la Recherche Scientifique (el primero, en 1947).

15. Se realizaron en el mes de julio de los años 1952, 1954, 1956 y 1958. El listado de ponentes se puede consultar en: Institut de Paleontologia Miquel Crusafont. L'Obra Científica de Miquel 

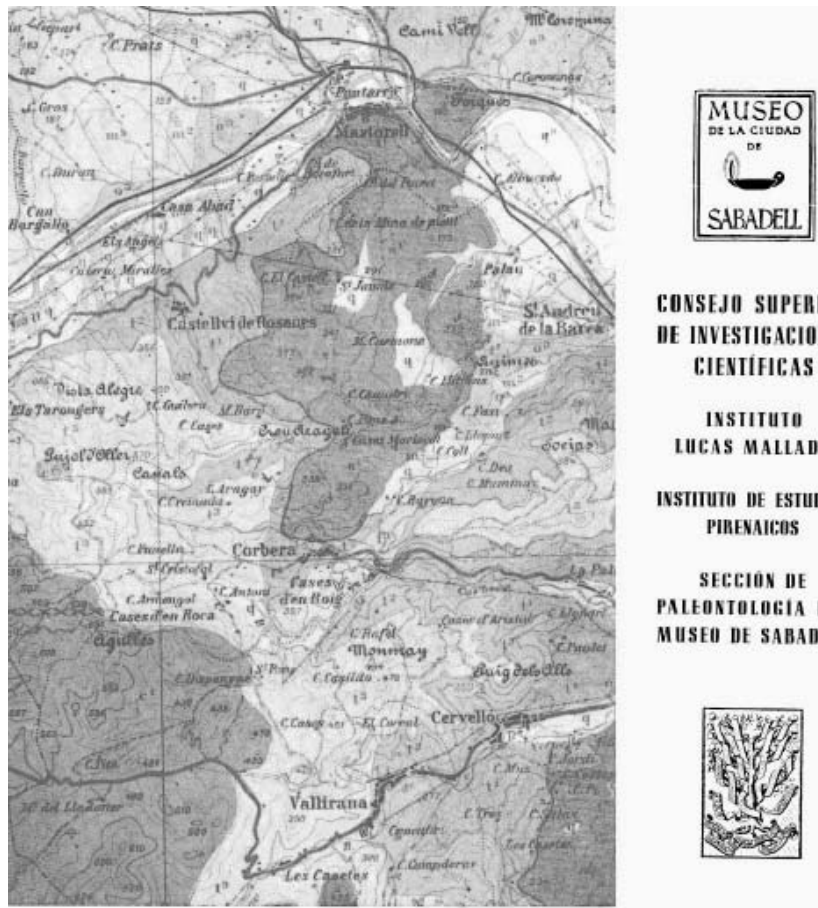

C.ONSEJU SUPERIOR

IE INVESTILACIONES

l:IENTIFICAS

INSIITUTE

LIIEAS MALLADA

INSTITUTก DE ESTIIIIIS

PIRENAIL:eS

SECLIUN UE

Paleastalotia oft

MUSEII DE SABADEUI

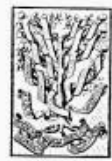

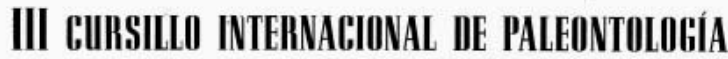

I R E U I I O I E L TERCIA RIO

4- 14 de Julio de 1950

TEMA CENTRAL.

Paleontología y Geología del Vallés-Penedés y de las cuencas terciarias pirenaicas

DIRECTOR: MIGUEL CRUSAFONT PAIRÓ

Figura 1. Portada del tríptico que anunciaba el III Cursillo Internacional de Paleontología, 1956, organizado por Miquel Crusafont con el apoyo del Museo de Sabadell y el CSIC (Archivo Miquel Crusafont, caja AMC-76, Cursets, congressos, conferències [sin catalogar], ICP, Sabadell).

Crusafont i Pairó (Sabadell, 1910-1983). Sabadell: Patronat dels Museus Municipals de Sabadell; 1993, p. 7-9. 
Estos eventos lograron inusitado éxito científico y social a pesar de la pobre infraestructura con que contaba el Museo y el escaso patrocinio económico estatal ${ }^{16}$, pero gracias al apoyo de la ciudadanía e instituciones de Sabadell ${ }^{17}$.

Pero los extranjeros también llegaron interesados en las colecciones y en los yacimientos que Crusafont daba a conocer e invitaba a estudiar. Ante esta internacionalización de la Paleontología española, Crusafont actuó con firmeza para exigir a los investigadores foráneos que el material paleontológico quedara en España. Él mismo escribe, justificando una intervención suya no autorizada en la cantera Zalduendos (Atapuerca):

«si intervine fue para que no ocurra como ocurre en otros lugares de España en los que -con connivencia de los españoles- se está esperando que aparezca el hombre fósil para que lo estudien los americanos. Yo he servido a la ciencia española sin tolerar que se inmiscuyan en ella personas que vienen a ejercer un colonialismo científico que nos deshonra» ${ }^{18}$.

Este empeño por evitar el expolio no debe ocultar su interés por enriquecer las colecciones del Museo de Sabadell, hasta el punto de protagonizar algunos debates sobre la pertenencia de algunas piezas depositadas temporalmente para su estudio en el Museo u otras que Crusafont reclamaba para éste ${ }^{19}$.

16. Aunque Crusafont siempre consideró insuficiente la ayuda oficial proveniente de ministerios como el de Educación Nacional o del CSIC, ésta le permitió hacer estudios, viajes exploratorios y contratar personal de apoyo. A modo de ejemplo: en 1944 recibe una ayuda para realizar trabajos en el Terciario Continental de Logroño y Aragón, véase Villalta, J. F. de y Crusafont, M. Copia de carta a José Ma Albareda 18 Feb 1944, AMC, doc. 045a/CProf/4383; Villalta, J. F. de y Crusafont, M. Copia de carta a Maximinio San Miguel de la Cámara. 20 Mar 1944, AMC, doc. 045a/CProf/4392. En 1950 la Sección del Museo recibe la primera subvención por parte del CSIC; Meléndez, Bermudo. Carta a M. Crusafont. 15 Jul 1950, AMC, doc. 045C/CProf/4830. En 1953 le fue concedida otra, de 10.000 pesetas, para pagar durante un año un preparador de fósiles; en 1960 recibió otra personal para asistir al Congreso de Paleontología en Copenhague. Meléndez, Bermudo. Carta a M. Crusafont. 16 Jul 1960, AMC, doc. 067a/Cacad/10365.

17. Instituciones como la Caixa d'Estalvis de Sabadell, la Fundació Bosch i Cardellach, el Ayuntamiento de Sabadell, el Diari de Sabadell, pero sobre todo, a la tenacidad del Museo de esta ciudad.

18. Crusafont, M. Carta al Presidente del Grupo Espeleológico de Burgos. Conflicto por excavaciones en la cantera a Zalduendos (Atapuerca). 10 Feb 1966, AMC, doc. 048c/Cprof/6749. Según el artículo de Oliver Hochadel en este dossier, la idea del «Colonialismo científico» fue usada recurrentemente como un recurso retórico en el contexto paleontológico español del siglo XX. Sobre el material fósil legado por Crusafont, véase: Gelabert, Laura Celià. Gestió del Patrimoni Paleontològic (s. XIX-XXI). Aproximació històrica a partir de l'analisi de collleccions. Definició del marc conceptual i estudi de cas. Barcelona: Universidad de Barcelona; 2008, p. 112-118.

19. Son ejemplo de ellos, respectivamente, los casos de la tortuga de Arévalo del yacimiento de «El Lugarejo». (García, Julio. Carta a M. Crusafont. 18 Feb 1968. AMC, doc. 049b/Cprof/7210; 
En los años cincuenta y sesenta la gestión del patrimonio paleontológico era todavía un asunto errático en lo institucional y carente de una legislación específica ${ }^{20}$. Aprovechando esta situación, Crusafont se convirtió en intermediario entre los científicos foráneos y los yacimientos y colecciones paleontológicas españolas. Celoso de sus áreas de trabajo y de los yacimientos bajo su «jurisdicción» (especialmente los catalanes, aragoneses y baleares), suscribió acuerdos tácitos en torno a las nuevas investigaciones a desarrollar en ellos, aunque en algún caso suscitaron polémica. Crusafont actuó algunas veces en consonancia con las autoridades que le solicitaban asesoría $^{21}$, y otras por encima de ellas estableciendo un código personal sobre el uso y conservación de las muestras que se encontrasen en trabajos de campo. Obró hasta tal punto con autonomía que los investigadores internacionales le solicitaban los permisos correspondientes para adelantar excavaciones y estudios en la península, y él los otorgaba sin oficialidad alguna $^{22}$. Finalmente tuvo que someterse a la legalidad vigente ${ }^{23}$.

Está claro que Crusafont edificó su obra en torno a las colecciones del Museo, pues era consciente de su importancia como elementos articulado-

Ministerio de Educación Nacional. Nota a: M. Crusafont. 7 Nov 1968, AMC, doc. 049b/ Cprof/7328), y del mastodonte de Polinyà; en el Arxiu Miquel Crusafont se encuentran al menos 18 documentos al respecto, entre cartas y recortes de prensa, el primero del 15 de octubre de 1965, AMC, doc. 068b/Cacad/11197 y el último del 18 de diciembre de 1965, AMC, doc. 048b/Cprof/6688.

20. García Fernández, Javier. La regulación y la gestión del Patrimonio Histórico-Artístico durante la Segunda República (1931-1939). Revista Electrónica de Patrimonio Histórico [publicación seriada en Internet]. 2007; 1: 1-43. [Disponible en: www.revistadepatrimonio.es/revistas/numero1/legislacion/estudios/articulo.php]; Díaz-Andreu, Margarita; Ramírez Sánchez, Manuel E. La Comisaría General de Excavaciones Arqueológicas (1939-1955). La administración del patrimonio arqueológico en España durante la primera etapa de la Dictadura Franquista. Complutum. 2001; 12: 325-343; Morales Romero, Jorge; Azanza Asensio, Beatriz; Gómez Ruíz, Elena. El Patrimonio Paleontológico Español. Coloquios de Paleontología. 1999; 50: 53-62.

21. Crusafont M. Copia de carta al: Comisario Provincial de Excavaciones Arqueológicas (Barcelona). 5 Ene 1951, AMC, doc. 045c/Cprof/4888.

22. Almela A. Nota a: Crusafont. 23 Sep 1964, 1964 AMC, doc. 048a/Cprof/6421; Febrer Carbó, Joaquín. Circular informativa a: M. Crusafont. 3 May 1965, AMC, doc. 068/Cacad/11116; Crusafont, M. Copia de carta a Walter G. Kühne (Berlín-Alemanya). Autorització per a recerca (excavacions per trobar microvertebrats) per part del Dr. Kühne i del seu alumne Sigfried Henkel. 10 Mar 1967, AMC, doc. 069a/Cacad/11621; López de Azcona, Juan Manuel (Comisión Nacional de Geología): Carta a M. Crusafont, adjuntando copia de nota sobre excavaciones sin permiso desarrolladas en Uña por el Dr. Henkel. 20 Dic 1969, AMC, doc. 049c/Cprof/7488.

23. Crusafont M. Copia de carta a José Manuel López de Azcona (Comisión Nacional de Geología); sol.licitud d'autorització per treballs paleontològics a Uña (Cuenca) a nom de Sigfried Henkel i Bernard Krebs (Freie Universität, Berlín). 18 Feb 1970, AMC, doc. 050a/Cprof/7526. 
res de la relación entre su labor y la sociedad, además de su vínculo con la comunidad científica (especialmente la extranjera). La gestión y el estudio del yacimiento de Can Llobateres (localizado en la Serra de Sant Iscle en Sabadell) ${ }^{24}$, es un ejemplo general de lo arriba mencionado, pero particular para el caso de la Paleoantropología ${ }^{25}$. Este yacimiento, fue estudiado por Crusafont a lo largo de muchos años, primero rudimentariamente, cuando era una cantera, pero después con especial intensidad entre 1960 y 1969, en asociación con el paleontólogo suizo Johannes Hürzeler, y con el patrocinio de la Wenner Gren Foundation for Anthropological Research de Nueva York y de la empresa Sandoz (que compró los terrenos) (Figura 2).

En este yacimiento, se han documentado hasta ahora más de 70 especies de mamíferos, además de restos de otros vertebrados e invertebrados ${ }^{26}$. Sin embargo, hasta 1969 se dieron a conocer tan solo una cuarentena de restos de interés paleoantropológico (la mayoría dientes aislados) ${ }^{27}$. Pero Can Llobateres guardó algunos de sus mejores tesoros para los discípulos y sucesores de Crusafont, especialmente el grupo dirigido por Salvador

24. Sobre la gestión de este yacimiento se adelanta una investigación del autor en colaboración con los Drs. Àngel Galobart y Bernat Vila, la cual ha sido expuesta preliminarmente en las Jornadas de la XXVII Sociedad Española de Paleontología (octubre de 2011), y en el Seminari d'Història de la Ciència: La història de la paleoantropología a Catalunya (Universitat Autònoma de Barcelona en Bellaterra, el 29 de abril de 2011).

25. La Paleoantropología era un asunto de vital interés en la cosmovisión de Crusafont, aunque no su especialidad. La abordó participando y organizando diversos eventos, entre otros: el Coloquio sobre «Paleontología y Evolución», organizado por Emiliano Aguirre en Madrid, Aguirre, E. Carta a M. Crusafont. 25 Oct 1959, AMC, doc. 046b/Cprof/5661; el cursillo «El hombre en la cumbre del proceso evolutivo» de la Junta de Cultura de Vizcaya (1961); el Curso sobre el «Evolucionismo y la Antropogénesis» en Montserrat, Crusafont, M. Copia de carta a Johannes Hürzeler. 16 Abr 1966, AMC, doc. 048/Cprof/6811; en tres cursillos de «Paleontología y Evolución humana» en 1970, 1971 y 1972 en el Instituto Provincial. Entre los escasos estudios se encuentran: Crusafont, Miquel. Los mamíferos, y en especial los Primates, del Eoceno prepirenaico. Notas y Comunicaciones del Instituto Geológico y Minero de España. 1965; 60: 127-140. Crusafont, Miquel; Golpe, Juana M. a New Pongids localities from the Miocene of Vallés-Penedés bassin (Catalonia, Spain). Journal of Human Paleontology. 1973; 2 (1): 17-24.

26. Alba, David M.; Casanovas-Villar, Isaac, Robles, J. M.; Marmi, Josep.; Moyà-Solà, Salvador. New paleontological excavations at the Late Miocene site of Can Llobateres 1 (Vallès-Penedès Basin, Iberian Peninsula): Resultados preliminares. In: Pérez-García, A.; Gascó, F.; Gasulla, J.; Escaso, F., eds. Viajando a Mundos Pretéritos. Morella: Ayuntamiento de Morella; 2011, p. 35-44.

27. Crusafont, Miquel; Hürzeler, Johannes. Les Pongidés fossiles d'Espagne. Comptes rendus de I'Académie des Sciences Paris. 1961; 252: 1-3. Crusafont, Miquel; Hürzeler, Johannes. Catálogo comentado de los Póngidos fósiles de España. Acta Geológica Hispánica. 1969; 4 (2): 44-48. 


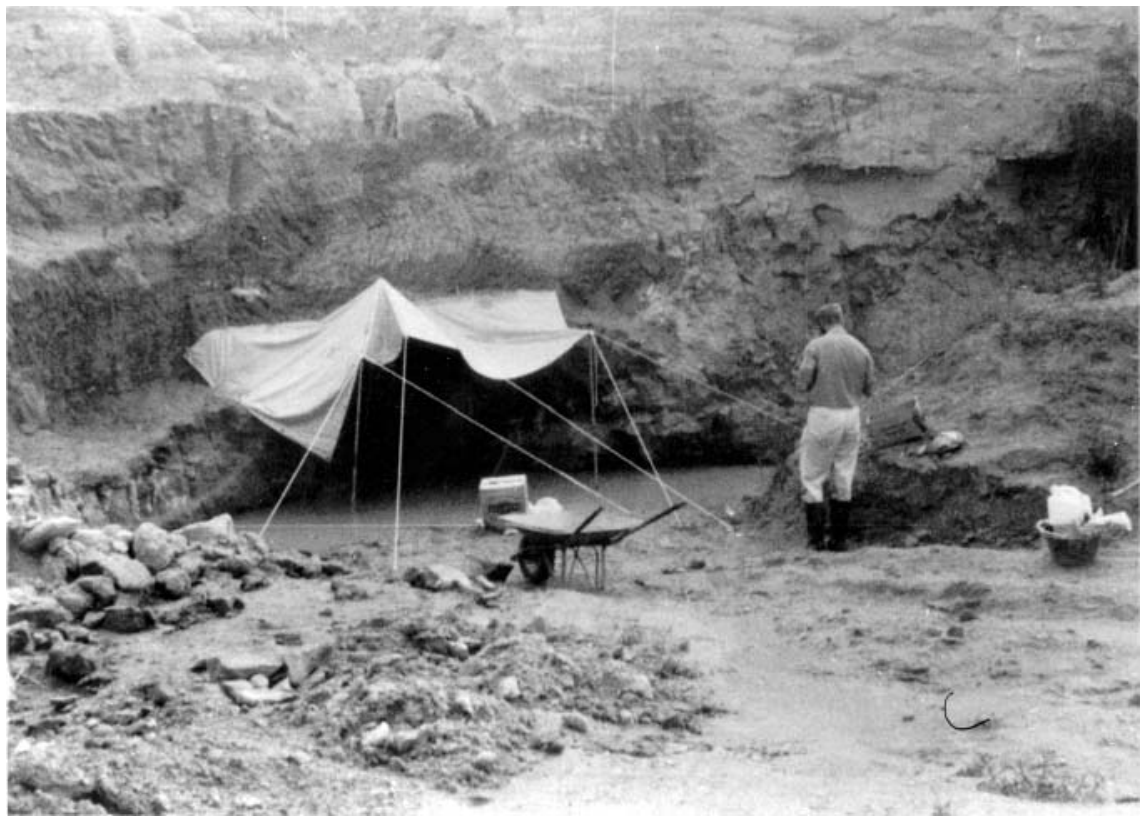

Figura 2. Excavaciones en el yacimiento Can Llobateres. Se aprecia al colaborador Narciso Sánchez en fecha sin definir entre 1960-1969 (Archivo Miquel Crusafont, Fotografies [sin catalogar], ICP, Sabadell).

Moyà-Solà (n. 1955), quienes generaron con sus descubrimientos un nuevo punto de inflexión de la Paleoantropología catalana e hicieron volver la mirada a Sabadell ${ }^{28}$, cuando el centro de la Paleoantropología española ya se había trasladado (desde los años ochenta) a Atapuerca, de la mano de Emiliano Aguirre (n. 1925), también discípulo de Crusafont.

28. Se destaca el hallazgo en este yacimiento de los restos de un homínido primitivo europeo, el Dryopithecus laietanus (1996) de 9,5 millones de años y denominado «Jordi», que junto con otros dos hallazgos del equipo de Moyà-Solà (esta vez en el yacimiento de Can Mata, Hostalets de Pierola), «Lluc» (Anoiapithecus brevirostis, 2009) de 11,9 millones de años, y «Pau» (Pierolapithecus catalaunicus, 2004) de 13 millones de años, conforman piezas claves en la explicación de la evolución humana en Europa. Según María Laura Moreno, estos primates aportan una simbología cultural nacional en la Cataluña democrática entre las décadas de 1990 y 2010. Moreno, María Laura. Los primeros catalanes. Jordi, Pau y Lluc, el influjo nacionalista en la búsqueda de los orígenes humanos. Barcelona: Universitat Autónoma de Barcelona-Universidad de Barcelona; 2010. 
Pero más allá de servir de vínculo, las colecciones le permitieron a Crusafont y a sus colaboradores tener mucho que decir. Esto resulta evidente si consideramos el número de sus publicaciones, que alcanzó 480, aunque solo 400 pueden considerarse «científicas» según los criterios actuales. La colaboración extranjera se vio reflejada también en las publicaciones, siendo escasa en los años cuarenta y cincuenta, pero mucho más frecuente en los años sesenta ${ }^{29}$.

A pesar de todo lo anterior, el alcance y la repercusión internacional de la obra de Crusafont fueron irregulares. Lo escrito por Simpson en la revista Evolution nos da idea de ello, pues lo señala como el «well-known Spanish palaeontologist», pero al mismo tiempo se queja del poco conocimiento de su obra debido a la ya mencionada restringida circulación de sus publicaciones, y reclama para ella mayor atención y utilización ${ }^{30}$. Sin embargo, lo logrado por Crusafont en relación a la internacionalización de la ciencia española y a su vinculación con escuelas extranjeras, fue un hecho poco común en la España de la época. Logró agruparse, es decir, trabajar al amparo del grupo que formó con los colegas finalistas-teístas españoles y europeos - la denominada, por el mismo Crusafont, «Escuela Meridional (...) ecléctica, más darwinista que lamarckista» ${ }^{31}-$, y codearse con colegas que no lo eran, entre ellos algunos de renombre como Simpson o Peter Robinson (n. 1932), del Museo de la Universidad de Colorado ${ }^{32}$,

29. En los años cuarenta y cincuenta los colaboradores de Crusafont fueron R. Lavocat y J. Viret; en los años sesenta trabajó especialmente con Gustav Heinrich Ralph von Koenigswald y Paul Sondaar (Utrecht), Johannes Hürzeler (Basilea), Louis Thaler y Jean Louis Hartenberger (Montpellier), Emille Heintz, A. Hoffstetter, Donald E. Russell, Germaine Petter, Leonard Ginsburg (París), Eric Delson y Björn Kurtén (Helsinki). Un recuento y análisis de la obra científica de Crusafont se ofrece en: Agustí, Jordi. L'obra palentològica de Miquel Crusafont. Ressenya bibliogràfica i trets fonamentals. 1994; 14, III época: 105-108; Truyols, n. 13, p. 35.

30. Simpson, George G. A review of masterometry. Evolution. 1965; 19: 249-255. Simpson se convirtió en amigo personal de Crusafont, y visitó España en 1969. Véase también Catalá, Jesús en este volumen.

31. En sentido estricto los finalistas no fueron darwinistas ya que el mecanismo de la evolución, la selección natural, no admitía idea alguna de finalidad y era antagónica a la Ortogénesis. De esta escuela formaban parte B. Meléndez y Emiliano Aguirre, los franceses J.P. Lehman, J. Viret, Lecompte de Nouy, J. Piveteau, los sacerdotes R. Lavocat y Frédéric-Marie Bergounioux, y los italianos Piero Leonardi y Alberto Carlo Blanc. Crusafont, Miquel. El tema de la evolución orgánica en España. Estudios Geológicos. 1951; 7 (13-14): 159-175 (166).

32. Robinson, Peter. Carta a M. Crusafont. Invitación a excavaciones en Túnez patrocinadas por la Smithsonian Institution. 28 May 1966, AMC, doc. 048/CProf/6834. 
para lo cual también empleó estrategias de conciliación y ambivalencia, como se verá a continuación.

\subsection{Estrategias de conciliación y ambivalencia}

La historia de la teoría de la evolución en España durante el franquismo nos ofrece un buen marco donde inscribir la actuación estratégica de Crusafont mediante la conciliación y la ambivalencia en algunas de sus posiciones ${ }^{33}$. Las siguientes palabras, escritas a Simpson, sirven de punto de partida a esta discusión:

«si aquí en España (...) nos metemos los científicos en honduras de tipo filosófico o religioso, lo hacemos para decir que según nuestro punto de vista nada hay en la ciencia que esté reñido con el Dogma. Esto es algo que nos vemos forzados a tratar, pues hablar de Evolución sólo desde el punto de vista científico, sería aquí casi imposible» ${ }^{34}$.

Está claro que los fundamentos ideológicos que -como creyente y científico- tenía Crusafont fueron tan fuertes que no renunció a ninguno de ellos, a pesar de la presión de contextos tan disímiles como la España

33. Sobre el evolucionismo en España durante el franquismo véase: Blázquez, Francisco. El evolucionismo en España y la síntesis neodarwinista (1939-1970). Madrid: UAM, Facultad de Filosofía y Letras; 2004. Blázquez, Francisco. La recepción del darwinismo en la universidad española (1939-1999). Anuario de Historia de la Iglesia (AHIg). 2009; 18: 55-68. Catalá, Jesús I. Cuatro décadas de historiografía del evolucionismo en España. Asclepio. 2009; 61 (2): 9-66; Florensa, Clara. Estudi del tractament de la teoria de l'evolució a La Vanguardia Española entre el 1939 i el 1978. Barcelona: Centre d'Història de La Ciència-Universitat Autònoma de Barcelona; 2010. Glick, Thomas F. El darwinismo en España en la primera mitad del siglo XX. Anthropos. 1982; 16: 76-81. Pelayo, n. 8.

34. Crusafont, M. Copia de carta a G. Simpson. 14 Oct 1953, AMC, doc. 045c/CProf/5009. Glick describe que Crusafont recubrió la evolución con una «pátina de retórica teológica». Glick, Thomas. The reception of the Synthetic theory in Spain. In: Engels, Eve-Marie; Glick, Thomas, eds. Miquel Crusafont and Teilhard de Chardin and the reception of the Synthetic theory in Spain. London/New York: Continuum; 2008, 553-568 (567). Sobre Paleontología, evolucionismo y religión en España durante el franquismo pueden verse: Blázquez, Francisco. A Dios por ciencia. Teología Natural en el franquismo. Asclepio. 2011; 63 (2): 453-476, con antecedentes de interés para el siglo XIX en: Pelayo, Francisco. La Paleontología. Un argumento para rebatir el darwinismo en el intento de armonizar ciencias naturales y religión. In: Hormigón Blánquez, Mariano, ed. Actas del Congreso de la Sociedad Española de Historia de las Ciencias, Vol. 1; Zaragoza: SEHCYT; 1984, p. 475-488; y Florensa, Clara, en este dossier. 
nacional-católica y la ciencia extranjera (al menos la percibida como positivista-materialista). Interesa aquí evitar el análisis presentista y sesgado, y resaltar que una posición intermedia y ambivalente le resultó conveniente, y que desde ella realizó intentos de conciliación, por ejemplo entre religión y ciencia ${ }^{35}$, y entre finalismo y neodarwinismo.

Dos ejemplos ilustran la posición que se le atribuye a Crusafont con respecto a las situaciones y conflictos arriba mencionados. El primero es el relacionado con Félix Rodríguez de la Fuente y su programa Planeta Azul. En 1971, Televisión Española - a petición del asesor religioso del Ministerio de Información y Turismo- censuró toda alusión directa o indirecta a la evolución en el programa ${ }^{36}$ (Figura 3). Crusafont se calificaba a sí mismo como un "perseguido», y alguien que tuvo que «luchar como gato panza arriba para defender el punto de vista de la Evolución», y en este caso lo hizo con vehemencia en contra de posiciones «enmohecidas, trasnochadas» ${ }^{37}$. Por un lado, dejó sentir todo su cientifismo diciendo que no existía ya científico naturalista alguno que fuera creacionista, y que la evolución era un hecho «archicomprobado». Por otro, afirmaba que «nunca la Ciencia, por "atrevida" que pueda parecer a determinadas mentes pusilánimes estará en contradicción con los dogmas de la fe cristiana católica» ${ }^{38}$.

35. Los trabajos más actuales sobre esta cuestión buscan comprender los personajes y situaciones en su contexto histórico, en lugar de justificarlos, ver por ejemplo: Ferngren, Gary. B. Science \& Religion. A historical introduction. Baltimore: Johns Hopkins University Press; 2002 (especialmente Wilson, David B. The historiography of Science and Religion, p. 13-27); Dixon, Thomas; Cantor, Geoffrey; Pumfrey, Stephen. Science and Religion. New historical perspectives. Cambridge: Cambridge University Press; 2010 (especialmente Dixon, Thomas. Introduction, 1-19).

36. Para seguir este caso véase: Acosta, Carlos. La teoría de la Evolución y la censura en TVE. Entre el fijismo, el finalismo ¿y el neodarwinismo? Actes d'Història de la Ciència i de la Tècnica. 2008; Nova Ėpoca 1 (1): 271-277.

37. Crusafont, M. Copia de carta a Félix Rodríguez de la Fuente. 10 Abr 1971, AMC, doc. 012c/ $\mathrm{CP} / 3034$.

38. Crusafont, M. Copias de cartas a: Director de la Revista Roca Viva y al Director adjunto de TVE. 9 Abr 1971, AMC, doc. 012c/CP/3032 y AMC, doc. 012c/CP/3033, respectivamente. Crusafont no fue el único que defendió la compatibilidad entre ciencia y religión, y que intentó eliminar la incompatibilidad de la dualidad católico-científico, tal es el caso del jesuita Antonio Romañá i Pujó (1900-1981); véase el trabajo de Vergara Torrente, Cristina. El debat cosmològic a l'Espanya de Franco. Barcelona: Universitat Autónoma de Barcelona-Universidad de Barcelona; 2012. Los problemas de compatibilidad entre la ciencia y el dogma católico en torno a temas como el origen de la vida, el evolucionismo y el origen del hombre, también fueron discutidos entre 1959 y 1961 en las tres primeras «Conversaciones intelectuales del 


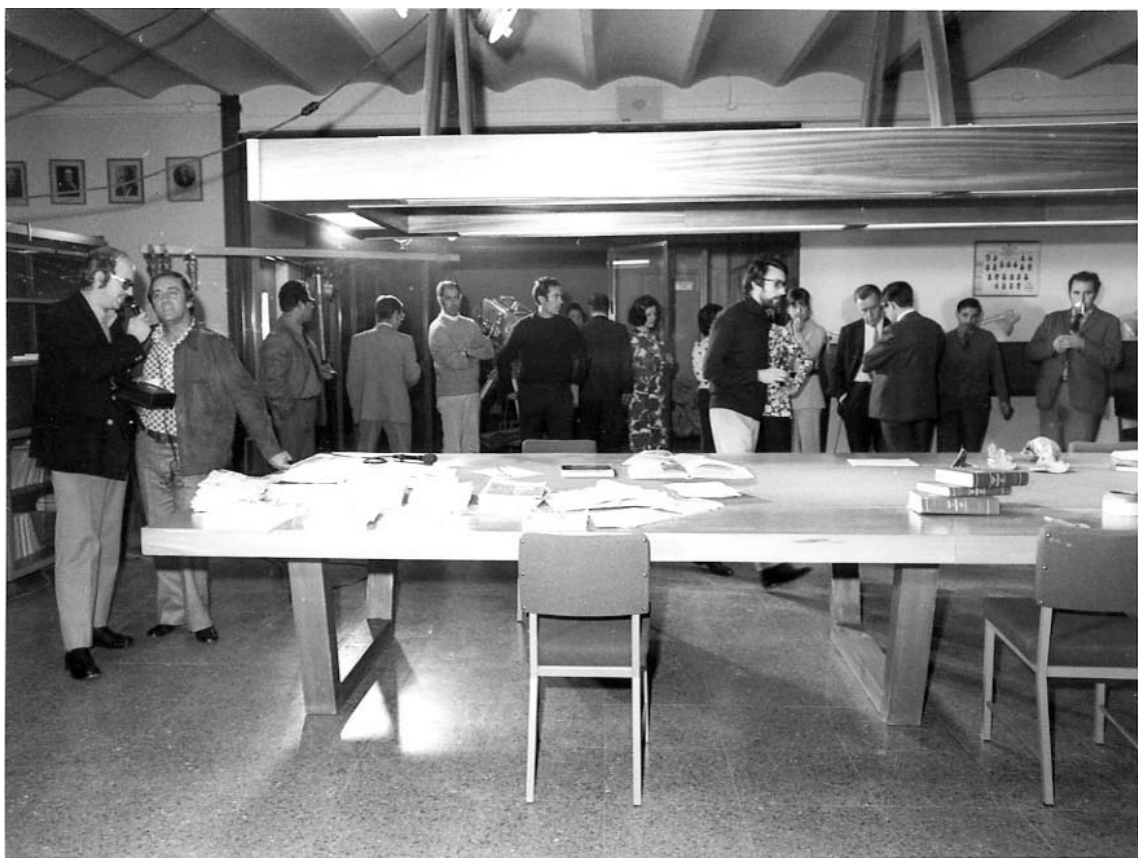

Figura 3. Visita de Félix Rodríguez de la Fuente al Instituto Provincial de Paleontología de Sabadell, con motivo de la filmación del programa «Planeta Azul» en 1970. Se aprecia en primer plano a Rodríguez de la Fuente siendo entrevistado, y al fondo — cuarto de derecha a izquierda - a Miquel Crusafont junto a su hija Anna (Archivo Miquel Crusafont, Fotografies [sin catalogar], ICP, Sabadell).

El segundo ejemplo está relacionado con su posición ante el neodarwinismo encarnado en su amigo Simpson, más concretamente con unas técnicas masterométricas ideadas por él y Jaume Truyols ${ }^{39}$, con cuyos resultados se pretendía la valoración cuantitativa del cambio evolutivo. Tanto Crusafont como algunos colegas también vieron en ellos pruebas de

Poblet», organizadas por la Sección de Cataluña y Baleares de la Asociación Menéndez Pelayo. Florensa, n. 33 y su contribución a este dossier.

39. Crusafont, M.; Truyols, J. A Biometric study of the evolution of fissiped carnivores. Evolution. 1956; 10 (3): 314-332. Crusafont, M.; Truyols, J. Estudios masterométricos en la evolución de los Fisípedos. I. Los módulos angulares alfa y beta. II. Los parámetros lineales P, C y T. Boletín del Instituto Geológico y Minero de España. 1957; 68: 89-224. 
la ortogénesis finalista ${ }^{40}$. Simpson, elogió lo técnico pero criticó las connotaciones espiritualistas en la revista Evolution ${ }^{41}$. Más allá de una nueva reseña del caso ${ }^{42}$, lo que interesa aquí es resaltar que Crusafont demostró tanto una conveniente ambivalencia como un sincero interés por conciliar posiciones más allá del ámbito de la amistad.

En 1965 le declara a Simpson que su objetivo era «tender un puente que ya me figuro que quizás será bastante frágil, pero puente al fin» y que, «ya verá Usted en mi próximo artículo como yo comparto la "criptoevolución" a que se refieren Ustedes los neo-darwinistas (yo también lo soy en su principio)», anuncio que cumplió, pero sin dejar de enfatizar la naturaleza antiazarosa de la selección natural ${ }^{43}$. Para acabar de ilustrar el asunto cabe citar las palabras de Crusafont a Simpson, en carta de 1969:

«es curioso, por lo demás, yo me siento neo-darwinista y sinteticista de la Evolución, es decir materialista, en cuanto se refiere a todo cuanto es inmanente en ella, pero no por ello - es decir, además - dejo de ser espiritualista y finalista. Caigo seguramente en el optimismo teilhardiano y en una ideología científica que quizás pueda parecer desorbitada dentro del positivismo de la ciencia ${ }^{44}$.

40. Simpson, G. Carta a Crusafont. 31 Mar 1965, AMC, doc. 048b/Cprof/6510, Utilización de J.P. Lehman del artículo de Crusafont en contra de la teoría sintética; Crusafont, M. Copias de cartas a: Fernando Riaza S. J. Pruebas de ortogenismo a partir de la masterometría. 28 Jun 1966, AMC, doc. 068c/Cacad/11388 y 28 Dic 1966, AMC, doc. 048c/Cprof/6946.

41. Simpson había realizado comentarios al método desde el mismo 1956 y luego en 1959. Simpson, G. Cartas a: M. Crusafont. 8 May 1956, AMC, doc. 046a/CProf/5272; 11 Feb 1959, AMC, doc.046b/Cprof/5549; 19 Mar 1959, AMC, doc. 046b/Cprof/5571.

42. Para seguir el caso: Glick, Thomas F. Miquel Crusafont i George Gaylord Simpson: Interferències Biogràfiques, confluències històriques. Arraona. 1994; 14. III época: 50-52; Agustí, n. 13, p. 34-35; y Catalá, Jesús I. Miquel Crusafont, George Simpson y la internacionalización de los estudios de Paleontología evolutiva en España, en este volumen.

43. Crusafont, M. Copias de cartas a: G. Simpson. 21 Abr 1965, AMC, doc. 048b/Cprof/6529; 14 Jun 1965, AMC, doc. 048b/Cprof/6566. El artículo prometido es: Crusafont, M. Neodarwinismo y ortogenismo: un intento de conciliación. Atlántida. 1965; 16: 394-401.

44. Crusafont, M. Carta a G. Simpson. 1 Abr 1969, transcrita por Glick, n. 42, p. 51. La cuestión ciencia y religión centrada en la participación de una divinidad en torno a la evolución no es un asunto zanjado, y sigue siendo tratado tanto en obras con perspectiva religiosa, como en otras en que se intenta conciliar posiciones mediante un compromiso constructivo de la teología con las ciencias, por ejemplo en: Russell, Robert John; Stoeger, William R., S.J.; Ayala, Francisco J., eds. Evolutionary and Molecular Biology. Scientific perspectives on divine action. II. Evolution and divine action. Vatican Observatory Publications. Vatican City State. Berkeley: Center for Theology and the Natural Sciences; 1998. 
Crusafont mantuvo encendido el debate finalismo-neodarwinismo durante los años sesenta y setenta, enfrentado a los biólogos moleculares y genetistas locales ${ }^{45}$, por ejemplo Ramon Margalef (1919-2004) y Antoni Prevosti (1919-2011) desde la Universidad de Barcelona, quienes habían ido desplazando a los geólogos como principales competidores de los paleontólogos, y a éstos como exclusivos referentes de la investigación y conocimiento de la evolución. Aunque venían pronunciándose desde finales de los cincuenta, los neodarwinistas (incluyendo algunos antiguos finalistas, como Emiliano Aguirre ${ }^{46}$ ) reavivaron el debate con la irrupción en la España de 1970 de Jacques Monod y su libro Azar y necesidad ${ }^{47}$.

En 1973, Crusafont enfermó de cáncer y en 1977 murió su esposa, acontecimientos que lo llevaron progresivamente a una pérdida significativa de su ímpetu y a situarse en un segundo plano del debate. En 1982, poco antes de su muerte, Crusafont recibiría «una gran distinció» al participar en un «curset coincidint amb l'aniversari de Darwin [en la inauguración del nuevo edificio de la Facultad de Biología de la Universidad de Barcelona] on prenien part eminents científics mundials», evento que marca un umbral final en el debate finalista-neodarwinista en España ${ }^{48}$.

En resumen, se concluye que la actuación ambivalente de Crusafont fue resultado de la interacción productiva entre sus convicciones científico-religiosas, las condiciones de posibilidad que le brindaba el contexto

45. Para una historia de la Biología y la Biología molecular en España: Calvó-Monreal Xavier. La Biologia molecular a l'Escola d'Enginyers: el Departament de Química Macromolecular. Quaderns d'Història de l'Enginyeria. 2006; 7: 45-72; Calvó-Monreal, Xavier. Polímeros e instrumentos. De la Química a la Biología molecular en Barcelona (1958-1977). Estudios sobre la Ciencia. Madrid: CSIC; 2012; Gomis, Alberto; Josa, Jaume. Veinticinco años de Historia de la Biología en España (1977-2002). Llull. 2003; 26: 109-156; Santesmases, María Jesús; Muñoz, Emilio. Una introducción al origen internacional de la comunidad científica española de Biología molecular. Arbor. 1994; 148 (583): 9-30; Santesmases, María Jesús; Muñoz, Emilio. The scientific periphery. In: Spain: The establishment of a biomedical discipline at the Centro de Investigaciones Biológicas, 1956-1967. Minerva. 1997; 35: 27-45.

46. Blázquez, 2009, n. 33, p. 63-64.

47. Monod, Jacques. Azar y necesidad. Ensayo sobre la filosofía natural de la biología moderna. Barcelona: Barral Editores; 1970.

48. Crusafont, M. Copia de carta a Lluís Tuca. 10 Ene 1983, AMC, doc. 052/Cprof/8593. Véase también Glick, n. 34, p. 567-568. El autor adelanta una investigación sobre los debates en torno a evolución en España durante el último franquismo y la transición, cuyos resultados parciales (identificación y documentación de siete debates con la participación de Crusafont) han sido expuestos en el seminario «La projecció pública del Darwinisme a Catalunya. Una aproximació», Centre d'Història de la Ciència, Universitat Autònoma de Barcelona, octubre de 2009. 
socio-político español, y la necesidad de pervivir en él ${ }^{49}$. Es decir, le sirvió para no ser represaliado por el régimen ${ }^{50}$, e incluso para ser apoyado por éste; para mantenerse en el grupo científico en el que se sentía cómodo y reconocido; para no quedar excluido de la corriente contemporánea que la disciplina seguía con fuerza en aquel momento, el neodarwinismo, y del que, además, Simpson - uno de sus principales valedores internacionalesera principal representante ${ }^{51}$.

\section{La institucionalización. De la Sección de Paleontología del Museo de Sabadell al Institut Català de Paleontologia (ICP)}

Crusafont realizó su labor científica desde la Sección de Paleontología del Museo de Sabadell (entre 1931 y 1969) y desde el Instituto Provincial de Paleontología (entre 1969-1983). Desde 1960 ejerció la cátedra universitaria, primero en la Universidad de Oviedo (1960-1963) y luego en la Universidad de Barcelona hasta su jubilación anticipada en 1980.

Al principio de su carrera, Crusafont - un farmacéutico sin título de naturalista - necesitaba de una institución que le respaldara, y ésta fue el Museo de Sabadell, cuya primera sala dedicada a la Paleontología fue in-

49. El trabajo clásico de Paul Forman sobre la influencia del medio social en la labor y posicionamiento ideológico de un científico ha sido recientemente cuestionado. Wise, M. Norton. Forman reformed, again. In: Carson, Cathryn; Kojevnikov, Alexei; Trischler, Helmuth, eds. Weimar culture and Quantum Physics: Selected papers by Paul Forman and contemporary perspectives on the Forman thesis. London/Singapore: World Scientific; 2011, p. 415-431.

50. Terminada la Guerra Civil (1939), Crusafont fue sometido a juicio sumarísimo acusado de catalanista (había formado parte de Acció Catalana). Crusafont i Sabaté, n. 3, p. 74.

51. Simon Schaffer plantea para las ciencias duras que los científicos en sus observaciones y laboratorios locales tienen que establecer redes socio-técnicas y políticas de apoyo, mientras que al mismo tiempo actúan en redes de comunicación más grandes. Además ellos han de integrar prudentemente el conocimiento local y global. Shaffer, Simon. Exact sciences and colonialism: Southern India in 1900. In: Epple, Moritz; Zittel, Claus, eds. Science as cultural practice. Volume I: Cultures and politics of research from the Early Modern period to the age of extremes. Berlin: Akademie Verlag; 2010, p. 121-139. Según Santesmases y Muñoz, el contexto político, tanto nacional como internacional, es útil para explicar la evolución de la creación de disciplinas como la Biología molecular y la Bioquímica, aunque sugieren que las relaciones internacionales y el apoyo nacional fueron necesarios, pero no suficientes para superar la distancia entre la comunidad científica española y los centros de desarrollo y productividad en tales disciplinas. Santesmases, María Jesús; Muñoz, Emilio. Scientific organizations in Spain (1950-1970): social isolation and international legitimation of biochemists and molecular biologists of the periphery. Social Studies of Science. 1997; 27 (2): 187-219. 
augurada en 1941, aunque Crusafont trabajaba con las colecciones de esta institución desde $1931^{52}$. En las décadas de los cuarenta y cincuenta invirtió gran esfuerzo en posicionar la Sección a escala nacional e internacional. Para ello requería integrarla en la estructura científica vigente en España, so pena de ser considerado simplemente un coleccionista de un museo de provincia. Además, tenía la necesidad de contar con un apoyo económico que el museo no podía ofrecerle.

Con estos objetivos Crusafont buscó el amparo del CSIC. En junio de 1949 solicitó al Consejo la creación de una sub-sección de Paleomastología o Paleobiología dependiente de la de Madrid ${ }^{53}$. El nombre de la propuesta Sección y la dependencia de la capital no eran cuestiones menores. La inminente organización de la Sección de Paleontología de Barcelona adjunta al CSIC a cargo de J. M. Bataller en el Museo Geológico del Seminario Conciliar de Barcelona - que se oficializaría en 1951- y el claro distanciamiento entre Bataller y Crusafont (y sus colaboradores) ${ }^{54}$ planteaban la dificultad de crear otra sección similar en la misma provincia, por lo que era necesario diferenciarlas suficientemente, como se verá más adelante.

La necesidad de contar con una Sección propia en Sabadell tomó tintes de urgencia en 1950, cuando Crusafont fue informado por Bermudo Meléndez de que el Patronato Alfonso El Sabio había acordado reemplazar a los «colaboradores» (con remuneración fija) por «ayudantes de laboratorio» en caso de poder mantener un horario fijo, o por «colaboradores honorarios» (sin sueldo ni gratificación fija) en caso de no poder tener tal dedicación. Esta situación tenía una incidencia directa sobre Crusafont, Villalta y Truyols, ya que, como Meléndez informó en la misma carta, Bataller no les había incluido como «colaboradores» de la nueva Sección de Barcelona y, por lo tanto, pasarían a ser solo «colaboradores honorarios». Meléndez matizó la

52. Crusafont, n. 2, p. 39.

53. Crusafont, M. Copias de cartas a: Maximino San Miquel de la Cámara. 4 Jun 1949, AMC, doc. 065c/Cadac/9308; 30 Jun 1949, AMC, doc. 065c/Cacad/9312; 9 Jul 1949, AMC, doc. 065C/ Cacad/9315; Copias de cartas a: José Ma Albareda (CSIC-Madrid). 9 mar 1951, AMC, doc. 065d/Cadac/9457; y a Bermudo Meléndez. 5 Abr 1951, AMC, doc. 065d/Cacad/9467.

54. Crusafont decía de Bataller que: «nada se puede esperar en cuanto espíritu de unión y de escuela [...] es un solitario científico y nosotros estamos convencidos de que se puede obtener mucho fruto de la colaboración, esto nos desespera». Crusafont, M. Copia de carta a Maximino San Miquel de la Cámara. 4 Jun 1949, AMC, doc. 065c/Cadac/9308. Otras alusiones a este respecto en: Crusafont, M. Copias de cartas a: Bermudo Meléndez. 5 May 1953, AMC, doc. 066a/ Cacad/9663; Truyols, Jaume. Carta a M. Crusafont. 27 May 1957, AMC, doc. 066c/ Cacad/10013. 
cuestión señalando la ventaja de no depender en adelante para nada de aquél y de Solé Sabarís. Profundamente afectado por esta situación Crusafont le responde que «tal como están las cosas muchas veces he pensado en el suicidio científico ¿para qué tanta lucha si uno se ve arrastrado por todas las corrientes sin esperanza de estímulos? ${ }^{55}$ »

A pesar de ello, Crusafont insistiría hasta lograr que en junio de 1951 su Sección también fuese integrada al CSIC, con el nombre de «Sección de Paleontología de Sabadell», adjunta al Instituto «Lucas Mallada», nombre que cambiaría en 1952 por el de «Sección de Paleobiología de Sabadell». El asunto del nombre tenía el objetivo inmediato de diferenciarla de la Sección de Barcelona pero, no solo nominalmente sino en el fundamento mismo de la Paleontología como actividad científica y profesional, acercándola más a la Biología (ya que la consideraba como una disciplina de las Ciencias de la Vida ${ }^{56}$ ), y en contra de la subordinación a la Geología como una herramienta meramente descriptiva ${ }^{57}$. Crusafont escribió al respecto que:

«Voy a hacer la solicitud del cambio de nombre de la Sección [de Paleontología a Paleobiología] pues esta disciplina tan desarrollada en otros países aquí no es casi tenida en cuenta y quizás sea esto el germen de posibles repercusiones oficiales en este sentido (para dentro de algunos años, naturalmente) que a todos los paleontólogos no puramente descriptivos nos interesan ${ }^{58}$.

En 1963, habiendo asumido la cátedra de Paleontología de la Universidad de Barcelona (después de la muerte de J. M. Bataller), se produjo

55. Meléndez, B. Carta a M. Crusafont. 26 Nov 1950, AMC, doc. 045c/CProf/4841; Crusafont, M. Copias de cartas a: Bermudo Meléndez. 15 Dic 1950, AMC, doc. 045c/CProf/4844.

56. Su posición al respecto quedó plasmada en: Crusafont, M. Paleontología y Biología: análisis y síntesis. Boletín Informativo del Instituto Provincial de Paleontología. 1972; 4 (2-3): I-V.

57. Crusafont, M. Copia de carta a Oriol Riba. 31 Dic 1968, AMC, doc. 049b/Cprof/7352. Esta postura en torno a la posición de la Paleontología frente a la Biología y a la Geología se prolongaría en el tiempo y le ocasionarían problemas al frente de la cátedra de la Universidad de Barcelona (que asumiría en 1962). Crusafont describe su situación en la cátedra como muy incómoda. Por un lado, los geólogos decían que explicaba una Paleontología demasiado biológica, y que la inmensa mayoría creían que los fósiles no servían más que como documentos de datación. Por otro, los biólogos creían que se inmiscuía en un terreno que no era el suyo. Crusafont también criticó que en el plan de estudio de Biología (1970) no se incluyera la Paleontología. Barcelona: Societat Catalana de Biologia, Evolució; 1974, p. 136.

58. Crusafont, M. Copia de carta a B. Meléndez. 5 Abr 1951, AMC, doc. 065d/Cacad/9467. Otras comunicaciones sobre el asunto: Crusafont, M. Copia de carta al: Ministerio de Educación Nacional. 9 Mar 1951, AMC, doc. 065d/Cadac/9455; Albareda, José M.a (CSIC-Madrid). Carta a Crusafont. 6 Nov 1951, AMC, doc. 065d/Cacad/9509. 
un nuevo cambio en la denominación de la Sección de Sabadell, que pasó a llamarse «Sección de Paleobiología y Paleontología de BarcelonaSabadell», un nuevo paso para hacer de la ciudad del Vallès el centro de la Paleontología catalana de la época. Era clara la querencia de Crusafont por su ciudad natal. La correspondencia evidencia que su cuartel general no era el laboratorio en la Universidad de Barcelona sino la Sección en Sabadell ${ }^{59}$, a la que intentaba dotar cada vez de mayor entidad. A pesar de que en 1965 pasaría a ser adjunta del nuevo Instituto «Jaume Almera» de Investigaciones Geológicas del CSIC, Crusafont escribe que era necesaria su independización del resto del Museo de Sabadell, ya que «s'havia quedat curta i necessitava més ampli espai per tal de desenvolupar-se» ${ }^{60}$, y propuso la creación de un instituto de Paleontología con un museo donde exponer de manera digna, al gran público y a la comunidad científica toda la riqueza paleontológica catalana y española.

La propuesta de Crusafont tuvo eco entre los dirigentes políticos y económicos a nivel local y provincial, especialmente en el alcalde de Sabadell, Josep M. Marcet, en la Diputació de Barcelona, y en la Caixa d'Estalvis de Sabadell. El nuevo Instituto Provincial de Paleontología (con un museo) se inauguró el 30 de octubre de $1969^{61}$ (Figura 4). A la muerte de Crusafont (en 1983) el Instituto Provincial a cargo de la Diputación de Barcelona recibió su nombre, el cual mantiene actualmente, reorganizado como Institut Català de Paleontología «Miquel Crusafont», a cargo de la Generalitat de Catalunya.

59. La etapa de Crusafont como catedrático merecería un estudio particular ya que se insinúa como una de las más polémicas e interesantes del personaje. Según J. Truyols, y se confirma en algunas cartas: Fontboté, Josep Maria. Carta a M. Crusafont. 2 May 1959, AMC, doc. 067a/Cacad/10236. Crusafont se dejó seducir por la idea de entrar a la cátedra universitaria, más que por su vocación, motivado por algunos colegas y amigos, y por la necesidad de darle, a sus cincuenta años, continuidad y estabilidad a su labor. Truyols, J. Miquel Crusafont paleontòleg. Quadern de les Arts i de les lletres de Sabadell. 1984; 38, 47; Truyols, n. 13, p. 35.

60. Crusafont, n. 2, p. 43.

61. Sobre la gestación del Instituto véase: Santafé i Llopis, Josep V. Gestació de I'Institut de Paleontologia. Una esperança contra tota esperança. Quadern de les arts i de les lletres de Sabadell. 1984; monogràfic 38; 48-51. Aguirre, Emiliano. Crusafont, Sabadell i l'estudi dels Fossils. Arraona, Revista d'Història. 1994; 14: 19-27. Truyols, Jaume. Miquel Crusafont Pairó (1910-1983). Actas de 1a Real Sociedad Española de Historia Natural. 1985; 81: 37-55. 

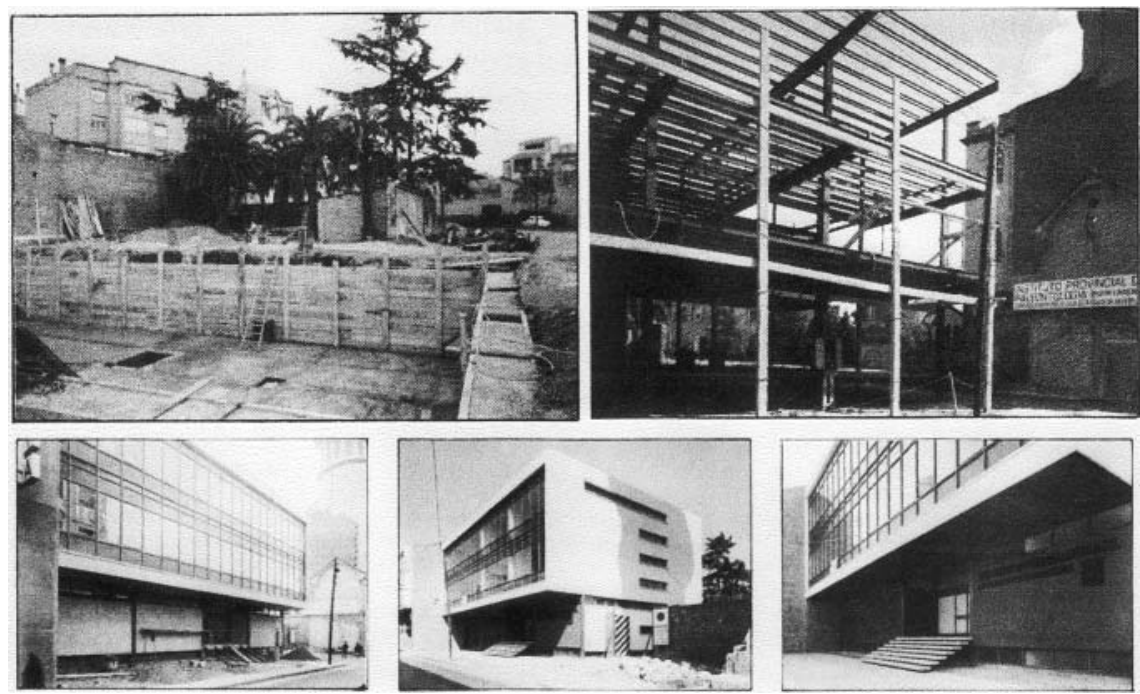

Figura 4. Secuencia de fotos de la construcción del Instituto Provincial de Paleontología en Sabadell, entre 1966 y 1969 (Archivo Miquel Crusafont, Fotografies [sin catalogar], ICP, Sabadell).

\section{Conclusiones}

Algunos de los paleontólogos españoles que trabajaron en las posguerras de mediados del siglo XX vencieron el aislamiento franquista. Es el caso de Miquel Crusafont quien actuó como un relaciones públicas para conformar redes profesionales amplias que le permitieron, desde una región periférica, dar visibilidad nacional e internacional a su colección, a su producción científica, y a su profesión.

A pesar de sus profundas convicciones ideológicas, Crusafont interactuó de forma productiva y utilitaria con los contextos culturales, políticos y científicos en que vivió, respondiendo a las condiciones de posibilidad (opciones y límites) que éstos generaban, con estrategias que le permitieron convertir a enemigos en aliados y viceversa, sin rupturas definitivas. Para ello utilizó a conveniencia la conciliación y la ambivalencia, lo que facilitó su trabajo ante el ojo censurador del régimen autoritario; le permitió trabajar bajo el amparo de un grupo suficientemente fuerte en el que parapetarse dentro y fuera de España (los colegas finalistas europeos); y lo mantuvo en la órbita de prestantes colegas que representaban nuevas corrientes de la disciplina, como el neodarwinismo de Georges Simpson. 
Como en un círculo virtuoso, las colecciones que Crusafont atesoró y estudió en el Museo de Sabadell y en el Instituto Provincial le permitieron tener algo original que decir, y lo que él y sus colegas dijeron de forma intensiva - aunque heterogéneamente recibido- produjo la sinergia necesaria para que, en torno a sus colecciones, se conformaran grupos de trabajo, se pusieran en marcha proyectos, se adecuaran espacios, se inauguraran salas de exhibición, se construyeran edificios, y se crearan entidades en las que se consolidó un ámbito de trabajo definido. Integró la Sección de Paleontología del Museo de Sabadell en el sistema científico vigente, otorgándole identidad y diferenciándola de otras entidades similares en la propia Cataluña, hasta convertirla en el reconocido centro de investigación que es hoy en día el Institut Català de Paleontologia. En él, al menos cuatro generaciones de paleontólogos y paleoantropólogos catalanes y españoles se han formado o han desarrollado su actividad investigadora (varios de ellos con valiosos resultados), apoyados en la defensa y la gestión que Crusafont - sin rehuir a la interacción productiva con los investigadores extranjeros- realizó del patrimonio paleontológico español, de las áreas de trabajo de los científicos locales, y de yacimientos como Can Llobateres. 


\section{One foot in heaven and the other in science. The productive interactions of Miquel Crusafont (1910-1983)}

Carlos Acosta Rizo . . . . . . . . . . . . . . . . . . . . . . . . . . . . . . . . . . . . .

1.--Introduction. 2.-Scientific emergence and survival of Miquel Crusafont. 2.1.-Creation of professional networks 2.2.-Strategies of conciliation and ambivalence. 3.-The institutionalization. From the Palaeontology section of the Sabadell Museum to the Catalonian Institute of Palaeontology. 4.-Conclusions.

ABSTRACT: After the Civil War and World War II (and even during it), and under very unfavourable conditions, some Spanish scientists resumed the dissemination of scientific practice and the Spanish palaeontological heritage. Both were of interest to foreign scientists, some of whom interacted with the Spanish researchers over the following decades. One of the latter group was the Catalan palaeontologist Miquel Crusafont Pairó (1910-1983) who engaged in intense professional activity, obtaining results that established him in the national scientific setting and gained him international recognition among colleagues with diverse scientific ideologies, while achieving a position for Spanish Palaeontology in Europe. This article analyzes some of the strategies adopted by Crusafont to overcome spatial and ideological differences and describes his particular activities around the defence and management of sites and collections. All of this allowed him to construct a research career from a province in a peripheral region under the particular conditions of Spain at that time and to lay the foundations of Palaeontology and Palaeoanthropology in contemporary Catalonia. 